\title{
On the Stochastic Modeling of COVID-19 under the Environmental White Noise
}

\author{
Shah Hussain, ${ }^{1}$ Elissa Nadia Madi ${ }^{D},{ }^{1}$ Hasib Khan $\left(D,{ }^{2}\right.$ Haseena Gulzar, ${ }^{3}$ Sina Etemad ${ }^{4},{ }^{4}$ \\ Shahram Rezapour $\mathbb{D}^{4,5}$ and Mohammed K. A. Kaabar $\mathbb{D}^{6,7}$ \\ ${ }^{1}$ Faculty of Informatics and Computing, Universiti Sultan Zainal Abidin (UniSZA), Besut Campus, Terengganu, Malaysia \\ ${ }^{2}$ Department of Mathematics, Shaheed Benazir Bhutto University, Sheringal, Dir Upper PO, 18000 Khyber Pakhtunkhwa, Pakistan \\ ${ }^{3}$ Department of Biotechnology, Shaheed Benazir Bhutto University, Sheringal, Dir Upper PO, 18000 Khyber Pakhtunkhwa, Pakistan \\ ${ }^{4}$ Department of Mathematics, Azarbaijan Shahid Madani University, Tabriz, Iran \\ ${ }^{5}$ Department of Medical Research, China Medical University Hospital, China Medical University, Taichung, Taiwan \\ ${ }^{6}$ Gofa Camp, Near Gofa Industrial College and German Adebabay, Nifas Silk-Lafto, 26649 Addis Ababa, Ethiopia \\ ${ }^{7}$ Institute of Mathematical Sciences, Faculty of Science, University of Malaya, Kuala Lumpur 50603, Malaysia
}

Correspondence should be addressed to Shahram Rezapour; rezapourshahram@yahoo.ca and Mohammed K. A. Kaabar; mohammed.kaabar@wsu.edu

Received 6 November 2021; Revised 14 February 2022; Accepted 18 February 2022; Published 8 March 2022

Academic Editor: Richard I. Avery

Copyright $\odot 2022$ Shah Hussain et al. This is an open access article distributed under the Creative Commons Attribution License, which permits unrestricted use, distribution, and reproduction in any medium, provided the original work is properly cited.

The COVID-19 pandemic has caused emotional loss to people around the world and provides an unusual test for public welfare, educational framework, food frameworks, and the world of work. The economic and social turmoil caused by this epidemic is increasing, and many people are at risk of falling into oppressive poverty. In this article, we describe the pandemic of infectious illness with the help of stochastic mathematical modeling. Based on the environmental white noise and by building appropriate Lyapunov functions and by applying Ito's formula, a few subjective properties are gotten. We provide a new mathematical model for the COVID-19 spread. The novel stochastic model is used to analyze the existence and prevalence of the disease, as well as its extinction. A numerical approach is developed for computing approximate solutions of the model. We show numerical simulations of deterministic and stochastic models of COVID-19 by utilizing the MATLAB software. In this direction, three graphs are included in the paper for the numerical interpretation of the stochastic model with the help of existing parametric and initial values for the model.

\section{Introduction}

A new virus known as "corona" was claimed to be wreaking havoc on the Chinese city of Wuhan in December 2019. This virus and its subsequent pandemic initially struck Wuhan and then spread to practically the entire world $[1,2]$. Hundreds of thousands of people died all across the world. It is difficult to come to a single conclusion on the virus's genesis. It could be the result of the migration patterns of people or a seafood market exchange. It is also possible that it is related to human-to-human relationships [3, 4]. So far, the virus has wreaked havoc on practically everything on the planet. Every aspect of human life has been badly impacted, including social life, health, the economy, and education. In battling the fatal pandemic, health researchers, government politicians, and health care authorities are perplexed. They all have a different perspective on the problem and are working hard to reduce the number of people who die as a result of the outbreak. For detail, one can see the useful work in $[5,6]$.

The coronavirus pandemic causes moderate respiratory symptoms in those who are affected. The symptoms of this illness include a fever, a dry cough, a throat infection, and exhaustion. Nasal infection, pains, and a sore throat are some of the other symptoms that people may experience. 
In order to describe the pandemic of infectious illnesses, mathematical modeling is an important technique. The goal of mathematical modeling is to use mathematical language to depict many types of real-world situations. The COVID19 was presented with the help of mathematical models in several recently published articles including a paper regarding coronavirus with two categories of asymptomatic and symptomatic individuals [7], a paper regarding the effect of quarantine on the spread of this disease [8], a paper in relation to this pandemic with deterministic structure [9], and a paper on the COVID-19 model with Haar wavelet collocation method [10]. Even, some researchers modeled this disease via different operators such as nonsingular derivatives [11] or Caputo singular derivatives [12].

A neural network is a type of nonparametric machine learning technique that is commonly used for prediction. New improved mathematical methods for predicting and analyzing pandemic casualties have been established by well-known researchers including Tutsoy et al. in [13, 14]. The nature of nonlinear dynamics is determined by the countries' health care infrastructure, as well as the qualities of enforced pharmacological or nonpharmacological policies. In the context of nonlinear dynamics, the neural network technique clearly improves the batch type leastsquares technique. In the case of linear dynamics, batch type least squares typically outperforms. Only when the casualties have less diversity, such as in intensive care and death casualties, the neural network produces more accurate findings [15]. In future, we will analyze our biological model with the help of batch type least squares and neural network approach which will provide us more real results only when the causalities have less variation.

In probability theory and associated fields, a stochastic or random process is a mathematical object, usually characterized as a group of random variables. In the 1930s, the first mathematical definition of a stochastic process as a family of random variables indexed by means of the change of the real line was given by Aleksandr Khinchin. Khinchin, Andrey Kolmogorov, Joseph Doob, and William Feller furthered the probability concept and stochastic modeling.

A stochastic model means to recognize the random nature of the input components. By contrast, in a stochastic model, the output is random in nature. The output is only a snapshot or an estimate of the characteristic of the model for a given set of inputs. A stochastic model will be run many times with the same output and gives distributions of the relevant results for a distribution of scenarios. The results of stochastic models require Monte Carlo simulations, although some stochastic models can have analytical solutions. Correlation can be important in stochastic models as they indicate the behavior of one variable is associated with that of the other. Stochastic models are more complex and difficult to interpret, so stochastic models require more expertise, expense, and computer power. A stochastic model has the capacity to handle uncertainties in the inputs applied. Stochastic models possess some inherent randomness-the same set of parametric values and initial conditions will lead to an ensemble of different outputs. In mathematics, the theory of stochastic processes is a signifi- cant aspect of probability theory and a subject of research for both theory and applications $[16,17]$. The word stochastic is used to portray different terms and objects in mathematics. For example, this includes a stochastic matrix, which describes a stochastic process known as a Markov process [18], and stochastic calculus [19, 20], which includes differential equations and integrals depending on stochastic processes such as the Wiener process, also called the Brownian motion (white noise) process [21-23].

In bioinformatics (in particular, in biochemical systems), there are two types of important white noises. These are intrinsic which could be seen as an inherent stochastic biochemical process and extrinsic white noise as cell-to-cell variability and external noise as environmental fluctuations, which in both cases can be viewed mathematically as white noise [24]

Also, stochastic modeling can be studied in various fields of science and engineering. In the financial markets, stochastic models are used to express stocks, relative stock price, commodities, and interest rates. In biological systems, adding stochastic "noise" to internal links for balance and other auditory interactions has been shown to assist boost. Manufacturing processes are thought to be random. For both continuous and batch manufacturing processes, this assumption is essentially valid. A process control chart illustrates a process control parameter over time and is used to record the monitoring and testing of the existing process. In the same way that events are interactions of systems, stochastic social science theory emphasizes unconscious processes. Because of the large number of variables involved, every event creates its own set of possibilities, making it unpredictable. Stochastic modeling of the dynamical problems is very much popular in the community of scientists. In their work, they have different aspects of their new models including the existence, extinction, and numerical simulations. For a detailed study of the stochastic modeling and their theoretical plus numerical analysis, we recommend the readers to read [25-27] and references therein.

In this article, we consider that the fluctuation of the $\beta$ and $\gamma$ is random and $\beta=\beta+\sigma_{1} d \mathscr{B}_{1}$ and $\gamma=\gamma+\sigma_{2} d \mathscr{B}_{2}$. With these considerations, we have the following new stochastic model for the COVID-19 spread.

$$
\left\{\begin{array}{l}
d \mathcal{S}^{*}=\left(b-k\left(1-\alpha_{0} \mathcal{S}^{*} \mathscr{I}^{*}\right)-\alpha_{0} k \beta \mathcal{S}^{*} \mathscr{I}^{*}-\mathrm{y} \mathcal{S}^{*}\right) d t-\sigma_{1} \alpha_{0} k \beta \mathcal{S}^{*} \mathscr{I}^{*} d \mathscr{B}_{1}, \\
d \mathscr{F}^{*}=\left(k\left(1-\alpha_{0} \mathcal{S}^{*} \mathscr{I}^{*}\right)+\alpha_{0} k \beta \mathcal{S}^{*} \mathscr{I}^{*}-\left(d_{0}+\gamma+\mathrm{y}\right) \mathscr{I}^{*}\right) d t+k \alpha_{0} \sigma_{1} \mathcal{S}^{*} \mathscr{F}^{*} d \mathscr{B}_{1}-\sigma_{2} \mathscr{I}^{*} d \mathscr{B}_{2}, \\
d \mathscr{R}^{*}=\left(\gamma \mathscr{I}^{*}-\mathrm{y} \mathscr{R}^{*}\right) d t+\sigma_{2} \mathscr{J}^{*} d \mathscr{B}_{2} .
\end{array}\right.
$$

Here, the symbol $\mathcal{S}^{*}$ represents the class of susceptible compartment, $\mathscr{I}^{*}$ represents the class of infected compartment, and $\mathscr{R}^{*}$ represents the class of recovered compartment from COVID-19. Also, $\alpha_{0}$ is the isolation rate, $\mathrm{y}$ is the natural death rate, $\gamma$ is the recovery rate, $\beta$ is the protection rate, $b$ is the birth rate, $k$ is the constant rate, and $d_{0}$ represents death due to corona. We have the following deterministic model [28]. 


$$
\left\{\begin{array}{l}
\frac{d \mathcal{S}^{*}}{d t}=\left(b-k\left(1-\alpha_{0} \mathcal{S}^{*} \mathscr{I}^{*}\right)-\alpha_{0} k \beta \mathcal{S}^{*} \mathscr{I}^{*}-\mathrm{y} \mathcal{S}^{*}\right) \\
\frac{d \mathscr{I}^{*}}{d t}=\left(k\left(1-\alpha_{0} \mathcal{S}^{*} \mathscr{I}^{*}\right)+\alpha_{0} k \beta \mathcal{S}^{*} \mathscr{I}^{*}-\left(d_{0}+\gamma+\mathrm{y}\right) \mathscr{I}^{*}\right. \\
\frac{d \mathscr{R}^{*}}{d t}=\left(\gamma \mathscr{I}^{*}-\mathrm{y} \mathscr{R}^{*}\right) .
\end{array}\right.
$$

In this article, we will present an analytical and computational study of the stochastic epidemic model (1) and will compare our results with the deterministic model (2) numerically for different parametric values for the prediction of future. Thus, this study can help the community in the awareness about the spread of the disease.

Partition of the paper. In this article, a stochastic COVID-19 model based on the environmental white noise is developed. In the second section, the essential requirement for the existence criteria, extinction, and persistence with the help of Lyapunov function is analyzed. A numerical approach is developed for computational and graphical analysis of the mentioned model which is included for the numerical interpretation of the stochastic model with the help of available parametric and initial values for the model in the third section.

\section{Existence of Solution}

For the existence of solution of our problem (1), we consider a general 3-dimensional stochastic ODE.

$$
d \mathbb{U}(t)=f(\mathbb{U}(t), t) d t+g(\mathbb{U}(t), t) d B(t),
$$

with $\mathbb{U}(0)=\mathbb{U}_{0} \in \mathbb{R}_{+}^{3}$ and define an operator $\mathscr{L}$ by

$$
\mathscr{L}=\frac{\partial}{\partial t}+\sum_{i=1}^{d} f_{i}(\mathbb{U}, t) \frac{\partial}{\partial \mathbb{U}_{i}}+\frac{1}{2} \sum_{i, j=1}^{3}\left[g^{\mathscr{T}}(\mathbb{U}, t) g(\mathbb{U}, t)\right]_{i j}
$$

By operating $\mathscr{L}$ on $\mathscr{V} \in C^{2,1}\left(\mathbb{R}^{d} \times\left[t_{0},+\infty\right]_{i} \mathbb{R}\right)$, then

$$
\begin{aligned}
\mathscr{L} \mathscr{V}(\mathbb{U}, t)= & \mathscr{V}_{t}(\mathbb{U}, t)+\mathscr{V}_{\mathbb{U}}(\mathbb{U}, t) f(\mathbb{U}, t) \\
& +\frac{1}{2} \operatorname{trace}\left[g^{T}(\mathbb{U}, t) \mathscr{V}_{\mathbb{U U}}(\mathbb{U}, t) g(\mathbb{U}, t)\right],
\end{aligned}
$$

in which $\mathscr{V}_{t}=\partial \mathscr{V} / \partial t, \mathscr{V}_{\mathbb{U}}=\left(\partial \mathscr{V} / \partial \mathbb{U}_{1}, \cdots, \partial \mathscr{V} / \partial \mathbb{U}_{d}\right)$, and $\mathscr{V}_{\text {UU }}=\left(\partial^{2} \mathscr{V} / \partial \mathbb{U}_{i} \partial \mathbb{U}_{j}\right)$. In the light of the Ito's formula, if $\mathbb{U}(t) \in \mathbb{R}^{3}$, we get

$$
d \mathscr{V}(\mathbb{U}(t), t)=\mathscr{L} \mathscr{V}(\mathbb{U}(t), t) d t+\mathscr{V}(\mathbb{U}, t) g(\mathbb{U}(t), t) d B(t)
$$

To prove the global positivity of solution for (1), we use Lyapunov analysis method. For this, consider $\Omega$ given by
$\Omega=\left\{\left(\mathcal{S}^{*}, \mathscr{I}^{*}, \mathscr{R}^{*}\right) \in \mathbb{R}_{+}^{3}: \frac{b}{\mathrm{y}+d_{0}} \leq \mathcal{S}^{*}+\mathscr{I}^{*}+\mathscr{R}^{*} \leq \frac{b}{\mathrm{y}}\right\} \subset \mathbb{R}_{+}^{3}$.

The following lemma gives the proof for the existence of solution of model (1).

Lemma 1. For $\left(\mathcal{S}^{*}, \mathscr{I}^{*}, \mathscr{R}^{*}\right)(0) \in \mathbb{R}_{+}^{3}$, one and one positive solution exists for (1) on $t \geq 0$ in $\mathbb{R}_{+}^{3}$ with probability equals to one.

Proof. Since (1) fulfills the local Lipschitz condition, then for $\left(\mathcal{S}^{*}, \mathscr{J}^{*}, \mathscr{R}^{*}\right)(0) \in \mathbb{R}_{+}^{3}$, we have $\left(\mathcal{S}^{*}, \mathscr{I}^{*}, \mathscr{R}^{*}\right) \in \mathbb{R}_{+}^{3}$ as a unique local solution on the semiopen interval $[0, \tau)$, in which $\tau$ is the explosion time. Now, our aim is to prove that $\tau=\infty$ for the global solution of (1). For this, we assume $k_{0}$ $\geq 0$ to be a very large number provided that $\left(\mathcal{S}^{*}, \mathscr{J}^{*}, \mathscr{R}^{*}\right)$ (0) lies in $\left[1 / k_{0}, k_{0}\right]$. For $k \geq k_{0}$, we define

$$
\begin{aligned}
\mathscr{I}^{*}{ }_{k}= & \inf \left\{t \in\left[0, \mathscr{I}^{*}{ }_{e}\right): \min \left\{\mathcal{S}^{*}(t), \mathscr{I}^{*}(t), \mathscr{R}^{*}(t)\right\}\right. \\
& \left.\left.\leq \frac{1}{k} \text { or } \max \left\{\mathcal{S}^{*}(t), \mathscr{I}^{*}(t), \mathscr{R}^{*}(t)\right\} \leq k\right\}\right\} .
\end{aligned}
$$

Let us assume that inf $\varnothing=\infty$. Since $\mathscr{T}^{*}{ }_{k}$ is increasing for $k \longrightarrow \infty$, let $\wp_{\infty}=\lim _{k \longrightarrow \infty} \wp_{k}$; then, we have $\wp_{\infty} \leq \wp_{e}$ a.s. Further, we need to investigate $\wp_{\infty}=\infty$ a.s. If this assertion is not valid, then there exist a constant $T>0$ and $\zeta(0,1)$ with $\mathbb{P}\left\{\wp_{\infty} \leq T\right\}>\zeta$. As a result, we have $k_{1} \geq k_{0}$ with $\mathbb{P}\left\{\wp_{k} \leq T\right\}$ $\geq \zeta, \forall k \geq k_{1}$. For $t \leq \wp_{k}$, we write

$$
\begin{aligned}
d\left(\mathcal{S}^{*}+\mathscr{I}^{*}+\mathscr{R}^{*}\right) & =\left[b-\mathrm{y}\left(\mathcal{S}^{*}+\mathscr{I}^{*}+\mathscr{R}^{*}\right)-d_{0} \mathscr{I}^{*}\right] d t \\
& \leq b-\mathrm{y}\left(\mathcal{S}^{*}+\mathscr{I}^{*}+\mathscr{R}^{*}\right) .
\end{aligned}
$$

This implies

$\left(\mathcal{S}^{*}+\mathscr{I}^{*}+\mathscr{R}^{*}\right)(t) \leq\left\{\begin{array}{l}{ }_{\frac{b}{y}} \text { if }\left(\mathcal{S}^{*}+\mathscr{F}^{*}+\mathscr{R}^{*}\right)(0) \leq \frac{b}{y}, \\ \mathcal{S}^{*}(0)+\mathscr{F}^{*}(0)+\mathscr{R}^{*}(0) \text { if } \mathcal{S}^{*}(0)+\mathscr{F}^{*}(0)+\mathscr{R}^{*}(0)>\frac{b}{y+d_{0}},\end{array}\right.$

where

$$
K=\max \left[\frac{b}{\mathrm{y}+d_{0}}, \mathcal{S}^{*}(0)+\mathscr{I}^{*}(0)+\mathscr{R}^{*}(0)\right] .
$$

We assume that

$$
\max \left\{\left|\mathcal{S}^{*}\right|,\left|\mathscr{I}^{*}\right|,\left|\mathscr{R}^{*}\right|,\right\}=\mathscr{M},
$$

for $\mathscr{M}>0$. We introduce a $\mathscr{C}^{2}$-function $\mathscr{V}: \mathbb{R}_{+}^{3} \longrightarrow \mathbb{R}_{+}$, by the following formulation:

$$
\begin{aligned}
\mathscr{V}\left(\mathcal{S}^{*}, \mathscr{I}^{*}, \mathscr{R}^{*}\right)= & \left(\mathcal{S}^{*}-1-\ln \mathcal{S}^{*}\right)+\left(\mathscr{I}^{*}-1-\ln \mathscr{I}^{*}\right) \\
& +\left(\mathscr{R}^{*}-1-\ln \mathscr{R}^{*}\right) .
\end{aligned}
$$


In the light of the Ito's formula, we get

$$
\begin{aligned}
d \mathscr{V}\left(\mathcal{S}^{*}, \mathscr{I}^{*}, \mathscr{R}^{*}\right) & \\
= & \mathscr{L} \mathscr{V}+\left[\sigma_{1}\left(\beta^{2}-\beta\right) k^{2} \alpha_{0}^{2} \mathcal{S}^{*} \mathscr{I}^{* 2}-\left(d_{0}+\gamma\right) k \alpha_{0} \sigma_{1} \mathscr{I}^{*} \mathcal{S}^{*}\right] \\
& \cdot d \mathscr{B}_{1}+\left(d_{0}+\gamma\right) \sigma_{2} \mathscr{I}^{*} d \mathscr{B}_{2},
\end{aligned}
$$

where

$$
\begin{aligned}
\mathscr{L} \mathscr{V}= & \left(1-\frac{1}{\mathcal{S}^{*}}\right) d \mathcal{S}^{*}+\left(1-\frac{1}{\mathscr{I}^{*}}\right) d \mathscr{I}^{*}+\left(1-\frac{1}{\mathscr{R}^{*}}\right) d \mathscr{R}^{*} \\
& +\sigma_{1}^{2} \alpha_{0}^{2} k_{2} \mathcal{S}^{* 2} \mathscr{I}^{* 2}+\sigma^{2} \mathscr{J}^{* 2} \\
= & \left(1-\frac{1}{\mathcal{S}^{*}}\right)\left(b-k\left(1-\alpha_{0} \mathcal{S}^{*} \mathscr{I}^{*}\right)\right)-\alpha_{0} k \beta \mathcal{S}^{*} \mathscr{I}^{*}-\mathrm{y} \mathcal{S}^{*} \\
& +\left(1-\frac{1}{\mathscr{I}^{*}}\right)\left(k\left(1-\alpha_{0} \mathcal{S}^{*} \mathscr{I}^{*}\right)+\alpha_{0} k \beta \mathcal{S}^{*} \mathscr{I}^{*}-\left(d_{0}+\gamma+\mathrm{y}\right) \mathscr{I}^{*}\right) \\
& +\left(1-\frac{1}{\mathscr{R}^{*}}\right)\left(\gamma \mathscr{I}^{*}-\mathrm{y} \mathscr{R}^{*}\right)+\sigma_{1}^{2} \alpha_{0}^{2} k_{2} \mathcal{S}^{* 2} \mathscr{I}^{* 2}+\sigma^{2} \mathscr{I}^{* 2} \\
\leq & b+\alpha_{0} \mathcal{S}^{*} \mathscr{I}^{*} k+k+\alpha_{0} k \beta \mathcal{S}^{*} \mathscr{I}^{*}+\gamma \mathscr{I}^{*} \\
\leq & b+\alpha_{0} \mathscr{M}^{3}+k+\alpha_{0} k \beta^{2} \mathscr{M}^{2}+\gamma \mathscr{M}:=\mathscr{K} .
\end{aligned}
$$

Therefore, we have

$$
\begin{aligned}
d \mathscr{V}(t)= & \mathscr{K} d t-\left[\sigma_{1}\left(\beta^{2}-\beta\right) k^{2} \alpha_{0}^{2} \mathcal{S}^{*} \mathscr{I}^{* 2}-\left(d_{0}+\gamma\right) k \alpha_{0} \sigma_{1} \mathscr{I}^{*} \mathcal{S}^{*}\right] \\
& \cdot d \mathscr{B}_{1}+\left(d_{0}+\gamma\right) \sigma_{2} \mathscr{J}^{*} d \mathscr{B}_{2} .
\end{aligned}
$$

Integrating (16) from 0 to $\tau \wedge \wp_{k}$ and taking expectation, we can get

$$
\begin{aligned}
\mathbb{E} \mathscr{V} & \left(\mathcal{S}^{*}\left(\tau \wedge \wp_{k}\right), \mathscr{I}^{*}\left(\tau \wedge \wp_{k}\right), \mathscr{R}^{*}\left(\tau \wedge \wp_{k}\right)\right) \\
& \leq \mathscr{V}\left(\mathcal{S}^{*}(0), \mathscr{I}^{*}(0), \mathscr{R}^{*}(0)\right)+\mathscr{K} \tau<\infty .
\end{aligned}
$$

Set $\Omega_{k}=\left\{\wp_{k} \leq t\right\}$ for $k \geq k_{1}$ where $\mathscr{P}\left(\Omega_{k}\right) \geq \zeta$; that is, $\forall \omega$ $\in \Omega_{k}$, and at least one of $\left(\mathcal{S}^{*}\left(\wp_{k}, \omega\right), \mathscr{I}^{*}\left(\wp_{k}, \omega\right), \mathscr{R}^{*}\left(\wp_{k}, \omega\right)\right)$ exists so that equals to $k$ or $1 / k$. Consequently,

$$
\begin{gathered}
\mathscr{V}\left(\mathcal{S}^{*}\left(\wp_{k}, \omega\right), \mathscr{J}^{*}\left(\wp_{k}, \omega\right), \mathscr{R}^{*}\left(\wp_{k}, \omega\right)\right) \\
\geq(k-1-\log k) \wedge\left(\frac{1}{k}-1+\log k\right),
\end{gathered}
$$

in which $a \wedge b$ stands for the minimum of $a$ and $b$. In view of (17) and (18), we have

$$
\begin{aligned}
& \mathscr{V}\left(\mathcal{S}^{*}(0), \mathscr{I}^{*}(0), \mathscr{R}^{*}(0)\right)+K \mathscr{T} \\
& \quad \geq \mathbb{E}\left[\mathscr{I}^{*} \Omega_{k} \mathscr{V}\left(\mathcal{S}^{*}\left(\wp_{k}, \omega\right), \mathscr{I}^{*}\left(\wp_{k}, \omega\right), \mathscr{R}^{*}\left(\wp_{k}, \omega\right)\right)\right] \\
& \quad \geq \zeta\left[(k-1-\log k) \wedge\left(\frac{1}{k}-1+\log k\right)\right],
\end{aligned}
$$

in which $\mathscr{I}^{*} \Omega_{k}$ stands for the indicator function of $\Omega_{k}$. By $k$ $\longrightarrow \infty$, we arrive at the following contradiction.

$$
\infty>\mathscr{V}\left(\mathcal{S}^{*}(0), \mathscr{I}^{*}(0), \mathscr{R}^{*}(0)\right)+K \tau=\infty
$$

Therefore, $\wp_{\infty}=\infty$ is valid. In view of system (1), we have

$$
\begin{gathered}
d \mathcal{S}^{*}+d \mathscr{I}^{*}+d \mathscr{R}^{*}=b d t-\mathrm{y}\left(\mathcal{S}^{*}+\mathscr{I}^{*}+\mathscr{R}^{*}\right) d t-\left(d_{0} \mathscr{I}^{*}\right) d t \\
d \mathcal{N}+y \mathcal{N}=b d t-\left(d_{0} \mathscr{I}^{*}\right) d t .
\end{gathered}
$$

Solving the above equation, we obtain that

$$
e^{\mathrm{y} t} \mathcal{N}=\frac{b}{\mathrm{y}} e^{\mathrm{y} t}-d_{0} \int_{0}^{t} e^{\mathrm{y} t} \mathscr{I}^{*} d t
$$

which implies that

$$
\mathcal{N} \leq \frac{b}{\mathrm{y}}
$$

On the other hand,

$$
d \mathscr{N}+y \mathcal{N}=b d t-\left(d_{0} \mathscr{I}^{*}\right) d t
$$

This implies

$$
d \mathscr{N}+y \mathcal{N} \geq b d t-\left(d_{0} \mathcal{N}\right) d t
$$

that is,

$$
d \mathcal{N}+\left(y+d_{0}\right) \mathcal{N} \geq b d t
$$

Solving the above inequality,

$$
\mathcal{N} \geq \frac{b}{\mathrm{y}+d_{0}}+\int_{0}^{t} \mathscr{N} e^{-\left(\mathrm{y} t+d_{0}\right) t} d t
$$

For the larger time, we have

$$
\mathcal{N} \geq \frac{b}{\mathrm{y}+d_{0}},
$$

and the proof is ended.

Theorem 2. Consider $\left(\mathcal{S}^{*}(t), \mathscr{I}^{*}(t), \mathscr{R}^{*}(t)\right)$ as the solution of the stochastic epidemic model (1). Then, the disease will die out exponentially with probability 1. In other words,

$$
\mathcal{S}^{*}(t), \mathscr{J}^{*}(t), \mathscr{R}^{*}(t) \longrightarrow 0 \text { and } \mathcal{S}^{*}(t) \longrightarrow b-t,
$$

as $t \longrightarrow \infty$. 
Proof. In the light of (1), we have

$$
\begin{aligned}
& d \log (Q)=\frac{1}{Q}\left[\left((d Q)+\sigma_{1} \alpha_{0} \beta k \mathcal{S}^{*} \mathscr{I}^{*}-\sigma_{1} \alpha_{0} \beta k \mathcal{S}^{*} \mathscr{I}^{*}\right) d \mathscr{B}_{1}\right. \\
& \left.+\left(\sigma_{2} \mathscr{J}^{*}-\sigma_{2} \mathscr{J}^{*}\right) d \mathscr{B}_{2}\right]+\frac{1}{2 Q^{2}}\left[\sigma_{1}^{2} \alpha_{0}^{2} \beta^{2} k^{2} \mathcal{S}^{* 2} \mathscr{J}^{* 2}\right. \\
& \left.+\sigma_{2}^{2} \mathscr{J}^{* 2}-\sigma_{1}^{2} \alpha_{0}^{2} \beta^{2} k^{2} \mathcal{S}^{* 2} \mathscr{J}^{* 2}-\sigma_{2}^{2} \mathscr{J}^{* 2}\right] d t \\
& =\frac{1}{Q}(d Q)\left(b-y \mathcal{N}-d_{0} \mathscr{I}^{*}\right) d t \text {. }
\end{aligned}
$$

Integrating (1) implies that

$$
\frac{Q-Q_{0}}{t}=\frac{1}{t}\left[\int_{0}^{t} \frac{b}{Q} d t-\mathrm{y} \int_{0}^{t} \mathcal{N} d t-d_{0} \int_{0}^{t} \mathscr{I}^{*} d t\right] .
$$

This gives

$$
\frac{Q}{t} \leq \frac{Q_{0}}{t}+\frac{1}{t} \int_{0}^{t} \frac{b}{Q} d t-\mathscr{H}(t),
$$

as $t \longrightarrow \infty$, and we have $Q \longrightarrow 0$. By putting $\mathscr{I}^{*}=0$ and $\mathscr{R}^{*}=0$ in (1), we have

$$
d \mathcal{S}^{*}+\mathrm{y} \mathcal{S}^{*} d t=(b-k) d t
$$

Solving this equation, we have

$$
\int_{0}^{t} d\left(e^{\mathrm{y} t} \mathcal{S}^{*}\right)=\int_{0}^{t} e^{\mathrm{y} t}(b-k) d t
$$

which yields

$$
\mathcal{S}^{*}=b-k\left(1-e^{-\mathrm{y} t}\right)+e^{-\mathrm{y} t} \mathcal{S}^{*}(0),
$$

and so

$$
\mathcal{S}^{*}=b-k \text { for } t \longrightarrow \infty \text {. }
$$

This ends the argument.

Theorem 3. For each arbitrary initial value $\left(\mathcal{S}^{*}(0), \mathscr{I}^{*}(0)\right.$, $\left.\mathscr{R}^{*}(0)\right) \in \mathbb{R}_{+}^{3}$, the disease is persistent and

$$
\left\langle\mathscr{I}^{*}\right\rangle \geq \frac{b}{\left(d_{0}+\gamma+y\right)}>0 .
$$

Proof. From system (1), we have

$$
d \mathcal{S}^{*}+d \mathscr{I}^{*}+d \mathscr{R}^{*}=b-\mathrm{y}\left(\mathcal{S}^{*}+\mathscr{I}^{*}+\mathscr{R}^{*}\right)-d_{0} \mathscr{I}^{*} .
$$

Integrating the above equation, we have

$$
\begin{aligned}
\frac{1}{t} & {\left[\mathcal{S}^{*}(t)-\mathcal{S}^{*}(0)+\mathscr{I}^{*}(t)-\mathscr{I}^{*}(0)+\mathscr{R}^{*}(t)-\mathscr{R}^{*}(0)\right] } \\
& \left.\left.\left.=b-\mathrm{y}\left(<\mathcal{S}^{*}>+<\mathscr{I}^{*}\right\rangle+<\mathscr{R}^{*}\right\rangle\right)-d_{0}<\mathscr{J}^{*}\right\rangle
\end{aligned}
$$

Also, integrating the third equation of (1), we obtain

$$
\frac{1}{t}\left[\mathscr{R}^{*}(t)-\mathscr{R}^{*}(0)\right]=\gamma<\mathscr{I}^{*}>-\mathrm{y}<\mathscr{R}^{*}>,
$$

which implies

$$
\mathrm{y}<\mathscr{R}^{*}>=\gamma<\mathscr{I}^{*}>-\frac{1}{t}\left[\mathscr{R}^{*}(t)-\mathscr{R}^{*}(0)\right] .
$$

From (27) and (28),

$$
\begin{aligned}
\mathscr{H}(t) & =b-\mathrm{y}<\mathcal{S}^{*}>-\mathrm{y}<\mathscr{I}^{*}>-\gamma \\
& <\mathscr{I}^{*}>+\frac{1}{t}\left[\mathscr{R}^{*}(t)-\mathscr{R}^{*}(0)\right],
\end{aligned}
$$

and also,

$$
<\mathcal{S}^{*}>=\frac{1}{\mathrm{y}}\left[b-\left(\mathrm{y}+\gamma+d_{0}\right)<\mathscr{J}^{*}>+\left(\mathscr{R}^{*}(t)-\mathscr{R}^{*}(0)\right)+\mathscr{H}(t)\right] .
$$

We construct a real-valued $\mathscr{C}^{2}$-function $\mathscr{V}$ on $\mathbb{R}$ by the following formulation.

$$
\mathscr{V}=\ln \mathscr{J}^{*}
$$

Thus,

$$
\begin{aligned}
d \mathscr{V}= & \frac{1}{\mathscr{I}^{*}}\left[\left(k\left(1-\alpha_{0} \mathcal{S}^{*} \mathscr{I}^{*}\right)+\alpha_{0} k \beta \mathcal{S}^{*} \mathscr{I}^{*}-\left(d_{0}+\gamma+\mathrm{y}\right) \mathscr{I}^{*}\right) d t\right. \\
& \left.+k \alpha_{0} \sigma_{1} \mathcal{S}^{*} \mathscr{I}^{*} d \mathscr{B}_{1}-\sigma_{2} \mathscr{J}^{*} d \mathscr{B}_{2}\right] \\
& -\frac{1}{2 \mathscr{I}^{* 2}}\left[k \alpha_{0} \sigma_{1} \mathcal{S}^{* 2} \mathscr{I}^{* 2}+\sigma_{2} \mathscr{I}^{* 2}\right] d t \\
= & \frac{k}{\mathscr{I}^{*}}-\alpha_{0} k \mathcal{S}^{*}(1-\beta)-\left(d_{0}+\gamma+\mathrm{y}\right)+\mathscr{B}^{*} .
\end{aligned}
$$

By integrating on both sides of above relation, we get

$$
\begin{aligned}
& \frac{1}{t}\left(\ln \mathscr{I}^{*}-\ln \mathscr{I}^{*}(0)\right)=\frac{1}{t} \int_{0}^{t} \frac{k}{\mathscr{I}^{*}} d t-k \alpha_{0}(1-\beta)<\mathcal{S}^{*}>-\left(d_{0}+\gamma+\mathrm{y}\right) \\
& =\frac{1}{t} \int_{0}^{t} \frac{k}{\mathscr{J}^{*}} d t-k \alpha_{0}(1-\beta)\left[\frac{b}{\mathrm{y}}-\frac{1}{\mathrm{y}}\left(d_{0}+\gamma+\mathrm{y}\right)+\mathscr{H}^{*}\right]-\left(d_{0}+\gamma+\mathrm{y}\right) \\
& \quad+\frac{1}{t} \int_{0}^{t} \mathscr{B}^{*} d t=\frac{1}{t} \int_{0}^{t} \frac{k}{\mathscr{J}^{*}} d t-k \alpha_{0}(1-\beta)\left[\frac{b}{\mathrm{y}}-\frac{1}{\mathrm{y}}\left(d_{0}+\gamma+\mathrm{y}\right)<\mathscr{I}^{*}>+\mathscr{H}^{*}\right] \\
& \quad-\left(d_{0}+\gamma+\mathrm{y}\right)+\frac{1}{t} \int_{0}^{t} \mathscr{B}^{*} d t=\frac{1}{t} \int_{0}^{t} \frac{k}{\mathscr{I}^{*}} d t-k \alpha_{0}(1-\beta) \frac{b}{\mathrm{y}} \\
& \quad+\frac{1}{\mathrm{y}}\left(d_{0}+\gamma+\mathrm{y}\right) k \alpha_{0}(1-\beta)<\mathscr{I}^{*}>-k \alpha_{0}(1-\beta) \mathscr{H}^{*}-\left(d_{0}+\gamma+\mathrm{y}\right) \\
& \quad+\frac{1}{t} \int_{0}^{t} \mathscr{B}^{*} d t .
\end{aligned}
$$




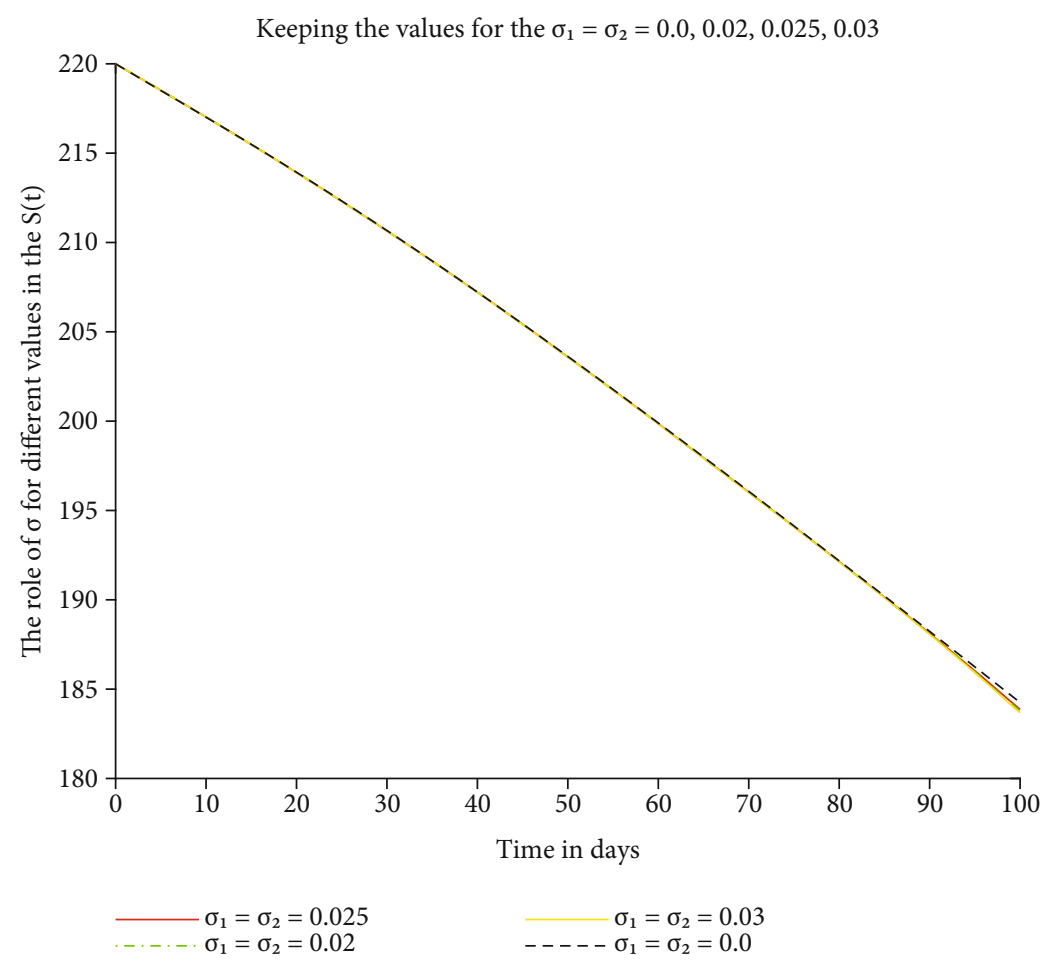

FIGURE 1: Joint solution of the given model (1) of COVID-19 for the order 1.0.

This further implies that

$$
\begin{aligned}
\frac{k}{\mathrm{y}} \alpha_{0}\left(d_{0}\right. & +\gamma+\mathrm{y})(1-\beta)<\mathscr{I}^{*}>\geq \frac{1}{t}\left(\ln \mathscr{I}^{*}-\ln \mathscr{I}^{*}(0)\right) \\
& -\frac{1}{t} \int_{0}^{t} \frac{k}{\mathscr{I}^{*}} d t+\frac{k}{\mathrm{y}} \alpha_{0}(1-\beta) b k \alpha_{0}(1-\beta) \mathscr{H}^{*} \\
& +\frac{1}{t} \int_{0}^{t} \mathscr{B}^{*} d t
\end{aligned}
$$

and for $t \longrightarrow \infty$, it becomes

$$
<\mathscr{J}^{*}>\geq \frac{b}{\left(d_{0}+\gamma+\mathrm{y}\right)}>0
$$

This ensures the persistence of the illness.

\section{Numerical Scheme and Results}

In this part of the research, we provide a numerical scheme for the suggested stochastic COVID-19 epidemic model (1). We have the parametric values $d_{0}=0.02, \beta=0.0009$ to 0.009 , $\alpha_{0}=0.0009$ to $0.009, \mathrm{y}=0.0062, b=10.7, k=0.00761, \gamma=$ $0.0003, \mathcal{S}^{*}(0)=220$ millions, $\mathscr{I}^{*}(0)=0$, and $\mathscr{R}^{*}(0)=0$.

This data was taken from the literature given in [28]. For the numerical results, we discretize the suggested stochastic mathematical model (1). The discretization yields the fol- lowing numerical scheme:

$$
\begin{aligned}
& \mathcal{S}^{*}{ }_{k+1}=\mathcal{S}^{*}{ }_{k}+\left(b-k\left(1-\alpha_{0} \mathcal{S}^{*}{ }_{k} \mathcal{Y}^{*}{ }_{k}\right)-\alpha_{0} k \beta \mathcal{S}^{*}{ }_{k} \mathcal{J}^{*}{ }_{k}-\mathrm{y} \mathcal{S}^{*}{ }_{k}\right) \Delta t \\
& -\sigma_{1} \alpha_{0} k \mathcal{S}^{*}{ }_{k} \mathcal{F}^{*}{ }_{k} \sqrt{\Delta t} \mathfrak{\wp}_{k}-\frac{\sigma_{1}^{2}}{2}\left(\alpha_{0} k \mathcal{S}^{*}{ }_{k} \mathcal{I}^{*}{ }_{k}\left(\mathfrak{\wp}_{k}^{2}-1\right)\right) \Delta t, \\
& \mathscr{F}^{*}{ }_{k+1}=\mathscr{F}^{*}{ }_{k}+\left(k\left(1-\alpha_{0} \mathcal{S}^{*}{ }_{k} \mathscr{I}^{*}{ }_{k}\right)+\alpha_{0} k \beta \mathcal{S}^{*}{ }_{k} \mathscr{Y}^{*}{ }_{k}-\left(d_{0}+\gamma+\mathrm{y}\right) \mathscr{F}^{*}{ }_{k}\right) \Delta t \\
& +\sigma_{1} \alpha_{0} k \mathcal{S}^{*}{ }_{k} \mathcal{I}^{*}{ }_{k} \sqrt{\Delta t} \wp_{k}+\frac{\sigma_{1}^{2}}{2}\left(\alpha_{0} k \mathcal{S}^{*}{ }_{k} \mathscr{I}^{*}{ }_{k}\left(\wp_{k}^{2}-1\right)\right) \Delta t \sigma_{2} \mathscr{J}^{*}{ }_{k} \wp_{k} \sqrt{\Delta t} \\
& -\frac{\sigma_{2}^{2}}{2} \mathscr{J}^{*}{ }_{k}\left(\wp_{k}^{2}-1\right) \Delta t \text {, }
\end{aligned}
$$$$
\mathscr{R}^{*}{ }_{k+1}=\mathscr{R}^{*}{ }_{k}+\left(\gamma \mathscr{F}^{*}{ }_{k}-\mathrm{y} \mathscr{R}^{*}\right) \Delta t+\sigma_{2} \mathscr{I}^{*}{ }_{k} \wp_{k} \sqrt{\Delta t}+\frac{\sigma_{2}^{2}}{2} \mathscr{I}^{*}{ }_{k}\left(\mathscr{\wp}_{k}^{2}-1\right) \Delta t .
$$

This scheme is utilized for the numerical results of the stochastic model (1). The numerical results are presented via three graphs which show a comparative analysis of the deterministic and stochastic models.

The graphical explanation of model (1) is given onward. The numerical simulations are presented with the help of the graphs. In the graphs, we have numerical data for the different values of the white noise $\sigma_{1}, \sigma_{2}$. In Figure 1, we have the susceptible class of model (1) for $\sigma_{1}=\sigma_{2}=0.0,0.02,0.025$, 0.03 . Figure 2 represents numerical data for the infection rate in the model as per given initial and parametric values while varying the white noise for $\sigma_{1}=\sigma_{2}=0.0,0.02,0.025$, 0.03 . Figure 3 shows simulations for the recovered class of model (1), changing the white noise for $\sigma_{1}=\sigma_{2}=0.0,0.02$, $0.025,0.03$. 

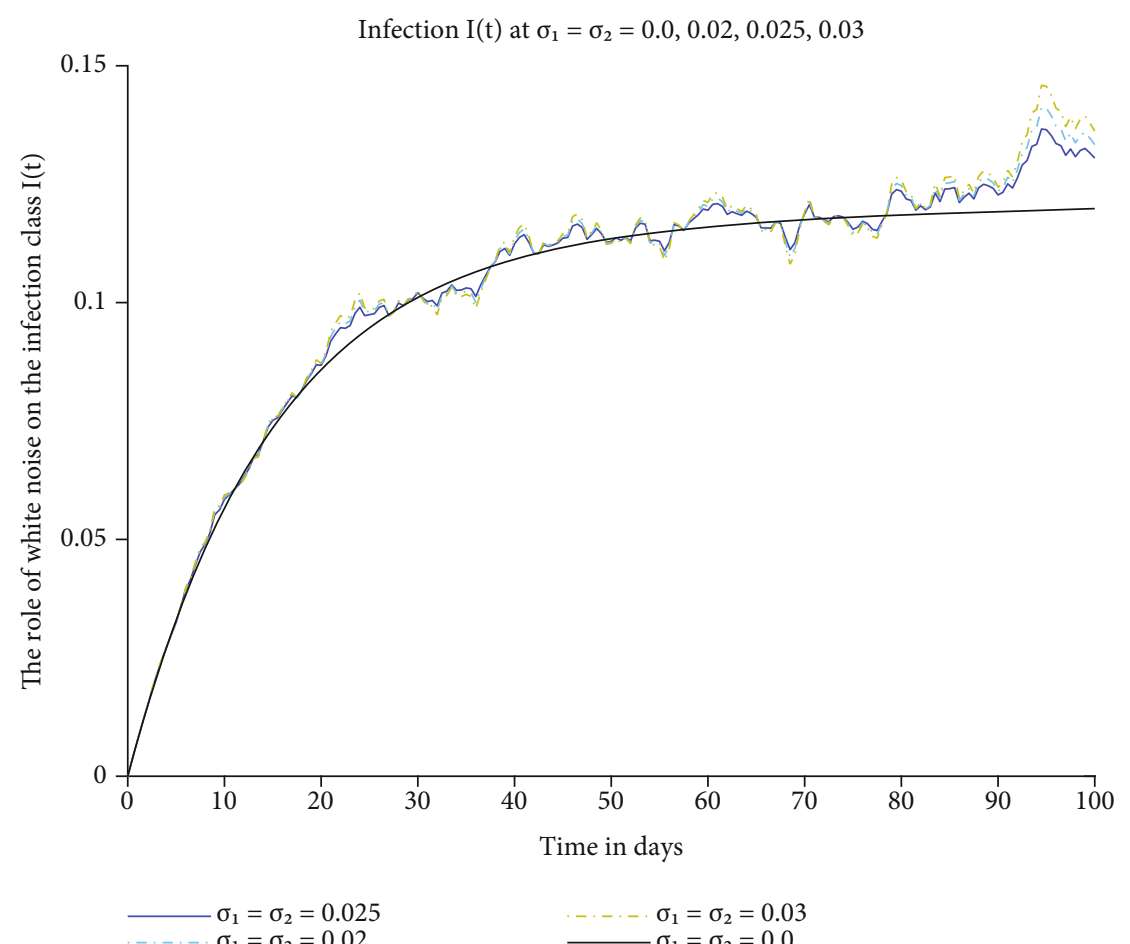

$\begin{aligned} \sigma_{1} & =\sigma_{2}=0.03 \\ \sigma_{1} & =\sigma_{2}=0.0\end{aligned}$

FIGURE 2: Joint solution of the given model (1) of COVID-19 for the order 0.99.

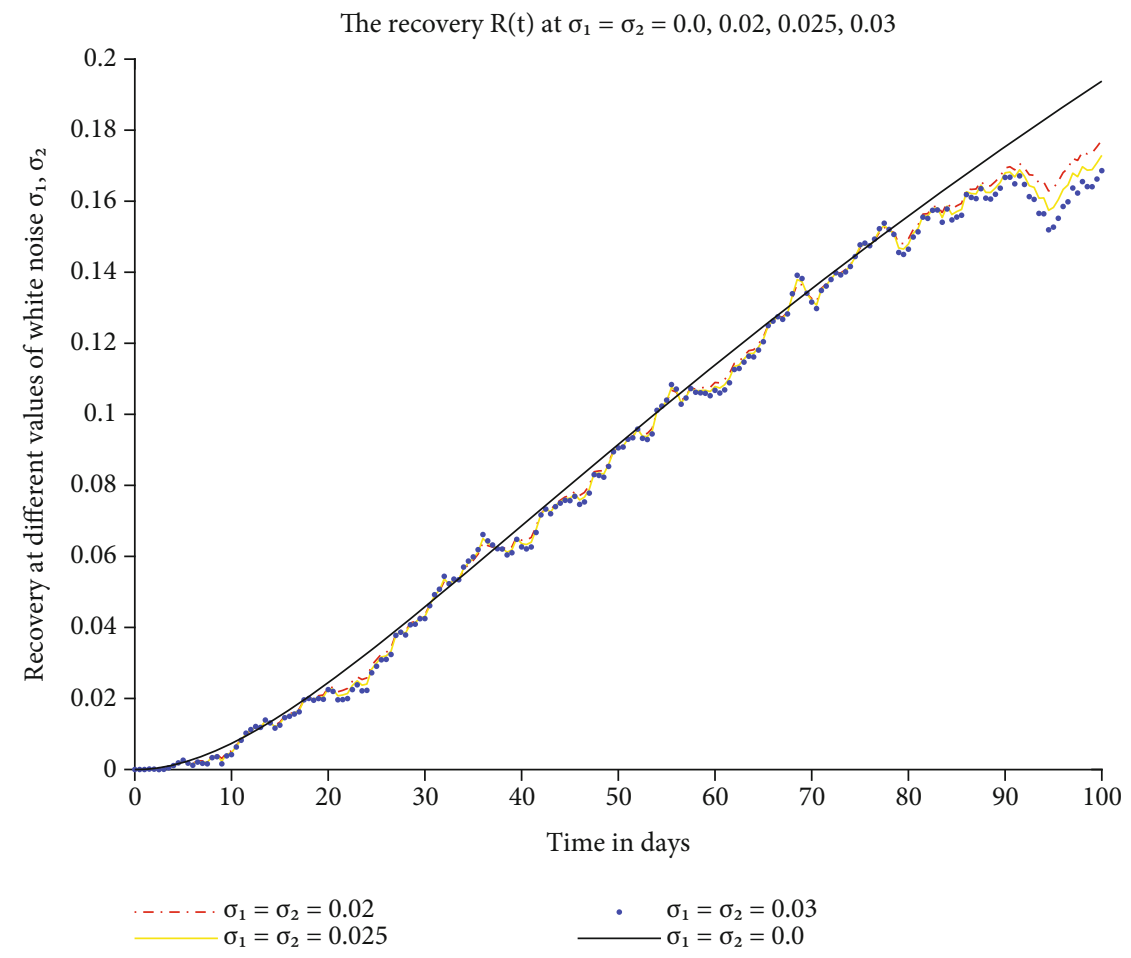

FIgURE 3: Joint solution of the given model (1) of COVID-19 for the order 0.98.

\section{Conclusions}

We provided and analyzed a novel stochastic mathematical model for the COVID-19 spread with white noise in this work. The model was mathematically examined for the exis- tence of a solution and other factors such as disease persistence and disease extinction. We presented the numerical scheme for the computational analysis of the model after the mathematical analysis, and the scheme was then tested for the available parametric and initial data in the literature. 
The computed findings clarified us with more realistic facts. Several diagrams were used to illustrate the numerical analysis. We mostly used two parameters in the graphs. We gave a graphical explanation of model (1) for different values of the white noise $\sigma_{1}, \sigma_{2}$. Also, We plotted all classes for different orders. The numerical simulations are presented with the help of the graphs. In the graphs, we have numerical data for the different values of the white noise $\sigma_{1}, \sigma_{2}$. In Figure 1, we have the susceptible class of model (1) for $\sigma_{1}=\sigma_{2}=$ $0.0,0.02,0.025,0.03$. Figure 2 represents numerical data for the infection rate in the model as per given initial and parametric values while varying the white noise for $\sigma_{1}=\sigma_{2}=$ $0.0,0.02,0.025,0.03$. Figure 3 shows simulations for the recovered class of model (1), changing the white noise for $\sigma_{1}=\sigma_{2}=0.0,0.02,0.025,0.03$. For future work as a continuation of the study, we aim to analyze model (1) in the fractional order settings.

\section{Data Availability}

No data were used to support this study.

\section{Conflicts of Interest}

The authors declare that they have no competing interests.

\section{Authors' Contributions}

The authors declare that the study was realized in collaboration with equal responsibility. All authors read and approved the final manuscript.

\section{Acknowledgments}

The fifth and sixth authors would like to thank Azarbaijan Shahid Madani University.

\section{References}

[1] A. Lee, "Wuhan novel coronavirus (COVID-19): why global control is challenging?," Public health, vol. 179, pp. A1-A2, 2020.

[2] Z. Du, L. Wang, S. Cauchemez et al., "Risk for transportation of coronavirus disease from Wuhan to other cities in China," Emerging Infectious Diseases, vol. 26, no. 5, pp. 1049-1052, 2020.

[3] S. Khan, G. Nabi, G. Han et al., "Novel coronavirus: how things are in Wuhan," Clinical Microbiology and Infection, vol. 26, no. 4, pp. 399-400, 2020.

[4] J. Riou and C. L. Althaus, "Pattern of early human-to-human transmission of Wuhan 2019 novel coronavirus (2019-nCoV), December 2019 to January 2020," Eurosurveillance, vol. 25, no. $4,2020$.

[5] C. Yang and J. Wang, "A mathematical model for the novel coronavirus epidemic in Wuhan," Mathematical biosciences and engineering: $M B E$, vol. 17, no. 3, pp. 2708-2724, 2020.

[6] P. Wu, X. Hao, E. H. Lau et al., "Real-time tentative assessment of the epidemiological characteristics of novel coronavirus infections in Wuhan, China, as at 22 January 2020," Eurosurveillance, vol. 25, no. 3, 2020.
[7] I. Ahmed, G. U. Modu, A. Yusuf, P. Kumam, and I. Yusuf, "A mathematical model of coronavirus disease (COVID-19) containing asymptomatic and symptomatic classes," Results in physics, vol. 21, article 103776, 2021.

[8] A. Babaei, M. Ahmadi, H. Jafari, and A. Liya, "A mathematical model to examine the effect of quarantine on the spread of coronavirus," Chaos, Solitons \& Fractals, vol. 142, article 110418, 2021.

[9] N. H. Tuan, V. V. Tri, and D. Baleanu, "Analysis of the fractional corona virus pandemic via deterministic modeling," Mathematicsl Methods in the Applied Sciences, vol. 44, no. 1, pp. 1086-1102, 2021.

[10] K. Shah, Z. A. Khan, A. Ali, R. Amin, H. Khan, and A. Khan, "Haar wavelet collocation approach for the solution of fractional order COVID-19 model using Caputo derivative," Alexandria Engineering Journal, vol. 59, no. 5, pp. 3221-3231, 2020.

[11] M. Sher, K. Shah, Z. A. Khan, H. Khan, and A. Khan, "Computational and theoretical modeling of the transmission dynamics of novel COVID-19 under Mittag-Leffler power law," Alexandria Engineering Journal, vol. 59, no. 5, pp. 3133$3147,2020$.

[12] N. H. Tuan, H. Mohammadi, and S. Rezapour, "A mathematical model for COVID-19 transmission by using the Caputo fractional derivative," Chaos, Solitons \& Fractals, vol. 140, article 110107, 2020.

[13] O. Tutsoy, A. Polat, S. Colak, and K. Balikci, "Development of a multi-dimensional parametric model with nonpharmacological policies for predicting the COVID-19 pandemic casualties," Ieee Access, vol. 8, pp. 225272-225283, 2020.

[14] O. Tutsoy, K. Balikci, and N. F. Ozdil, "Unknown uncertainties in the COVID-19 pandemic: multi-dimensional identification and mathematical modelling for the analysis and estimation of the casualties," Digital Signal Processing, vol. 114, article 103058, 2021.

[15] O. Tutsoy and A. Polat, "Linear and non-linear dynamics of the epidemics: system identification based parametric prediction models for the pandemic outbreaks," ISA transactions, 2021.

[16] J. L. Doob, Stochastic Processes, Wiley, New York, 1991.

[17] D. Williams, Probability with Martingales, Cambridge University Press, Cambridge, 1991.

[18] L. C. G. Rogers and D. Williams, Diffusions, Markov Processes and Martingales: Vol. 1, Foundations, John Wiley and Sons, Ltd., Chichester, UK, 2nd ed edition, 1994.

[19] Z. Zhang and G. Karniadakis, Numerical Methods for Stochastic Partial Differential Equations with White Noise, Springer, Cham, Switzerland, 2017.

[20] F. Bernal and J. A. Acebron, "A comparison of higher-order weak numerical schemes for stopped stochastic differential equations," Communications in Computational Physics, vol. 20, no. 3, pp. 703-732, 2016.

[21] F. Biagini, B. Oksendal, A. Sulem, and N. Wallner, "An introduction to white-noise theory and Malliavin calculus for fractional Brownian motion," Proceedings of the Royal Society of London. Series A: Mathematical, Physical and Engineering Sciences, vol. 460, no. 2041, pp. 347-372, 2004.

[22] K. Burdzy, Brownian Motion and its Applications to Mathematical Analysis, Springer, Cham, Switzerland, 2014.

[23] S. Hussain, E. N. Madi, H. Khan et al., "Investigation of the stochastic modeling of COVID-19 with environmental noise from the analytical and numerical point of view," Mathematics, vol. 9, no. 23, p. 3122, 2021. 
[24] X. Lei, W. Tian, H. Zhu, T. Chen, and P. Ao, "Biological sources of intrinsic and extrinsic noise in cI expression of lysogenic phage lambda," Scientific Reports, vol. 5, no. 1, article 13597, 2015.

[25] N. H. Sweilam, S. M. Al-Mekhlafi, and D. Baleanu, "A hybrid stochastic fractional order coronavirus (2019-nCov) mathematical model," Chaos, Solitons \& Fractals, vol. 145, article 110762, 2021.

[26] A. Babaei, H. Jafari, S. Banihashemi, and M. Ahmadi, "Mathematical analysis of a stochastic model for spread of coronavirus," Chaos, Solitons \& Fractals, vol. 145, article 110788, 2021.

[27] J. Danane, K. Allali, Z. Hammouch, and K. S. Nisar, "Mathematical analysis and simulation of a stochastic COVID-19 Levy jump model with isolation strategy," Results in Physics, vol. 23, article 103994, 2021.

[28] R. Ud Din and E. A. Algehyne, "Mathematical analysis of COVID-19 by using SIR model with convex incidence rate," Results in Physics, vol. 23, article 103970, 2021. 